\title{
Editorial
}

\section{Is the practice of haematology evidence based? I. Evidence based clinical practice}

There has been much attention focused on the application of evidence based medicine to the management of individual patients. Two recent editorials have also discussed the role pathologists may have in developing an evidence based approach to both diagnostic testing ${ }^{1}$ and morphological interpretation. ${ }^{2}$ Haematologists have various roles which include the clinical management of individual patients, selection and interpretation of results from diagnostic tests, and morphological interpretation of blood films and bone marrows. In this article we discuss whether an evidence based approach can be applied to haematologists' clinical practice. The areas of morphological diagnosis and laboratory testing will be covered in two subsequent articles. We recognise that haematologists have other roles, such as clinical liaison with colleagues. We will not cover this area since we believe that if the main clinical and laboratory areas are evidence based then so will the clinical liaison role. In addition, we will not cover the role a haematologist has in the management of laboratory departments.

The practice of clinical haematology in the United Kingdom has been characterised for many years by the participation of haematologists in multicentre randomised controlled trials, particularly in the areas of treatment of leukaemia and lymphoma. This process has to some extent helped to develop and promote the implementation of evidence based practice. Haematologists, however, treat quite a wide range of diseases and it is therefore worthwhile reviewing the definition of evidence based medicine.

Evidence based medicine has been defined as the conscientious and judicious use of current best evidence from clinical care research in the management of individual patients. ${ }^{3}$ This definition requires that evidence is applied consistently to each patient for whom it is relevant. This approach also requires the incorporation of the haematologist's clinical expertise which balances the risks and benefits of any possible treatment for each patient and takes into account the patient's clinical circumstances and preferences. This latter part of the definition is an extremely important part of the process and is often overlooked by those who believe evidence based medicine is a "cook book" approach to clinical practice.

The process of practising evidence based medicine involves five linked steps ${ }^{4}$ :

- To convert our information needs into answerable questions

- To track down with maximum efficiency the best evidence with which to answer these questions

- To critically appraise that evidence

- To apply the results of the appraisal to our clinical practice

- To evaluate our own performance.

Each of these steps is equally important and requires training and expertise in areas such as formulating clinical questions, review of published reports, critical appraisal skills, and so on. Using this approach, to what extent is the clinical practice of haematology evidence based?

In a study of a single haematology practice it has been shown that $70 \%$ of therapeutic interventions which were offered to patients over a one month period were evidence based. ${ }^{5}$ This experience in haematology compares with that of a previously reported study of inpatient general medicine, in which $82 \%$ of interventions were evidence based. ${ }^{6}$ Illustrative examples from the first study ${ }^{5}$ include evidence from randomised controlled trials for the use of CHOP (cyclophosphamide, adriamycin, vincristine, prednisolone) chemotherapy for the treatment of high grade non-Hodgkin's lymphoma. ${ }^{7}$ This level of evidence contrasts with the results of a systematic review of immune thrombocytopenia purpura. This study concluded that there was no evidence of an effect of glucocorticoid treatment on either morbidity or mortality of these patients. ${ }^{8}$ Despite this lack of evidence, the panel who produced the guidelines concluded that prednisolone treatment was appropriate in the management of significant bleeding episodes in patients with severe thrombocytopenia. It would now seem to be ethically unacceptable to perform a randomised placebo controlled trial of the use of prednisolone in this situation.

These types of studies do raise various questions regarding implementation of evidence based practice. One of the major blocks in developing an evidence based approach to clinical practice is the time required for review of published reports. For each clinical scenario generated, a complete literature review has to be undertaken. This can be quite time consuming even with electronic forms of searching such as Medline and the Cochrane library. In the study quoted above of one haematology practice, only three relevant systematic reviews or meta-analyses were found in the literature search. These systematic reviews were only directly relevant when assessing the therapeutic options available to a minority of patients. Therefore for most clinical scenarios original searches of published reports would have to be undertaken, together with critical appraisal of all the papers identified. There is also considerable duplication of effort each time the same search is undertaken by individual haematologists. Clearly this is one major obstacle to implementing evidence based practice in haematology. Another possible difficulty is that while there are many randomised controlled trials in the areas of treatment for leukaemia and lymphomas, there are few, if any, relevant trials in other common areas of haematology, such as immune thrombocytopenia. ${ }^{8}$ If these are some of the difficulties, how can they be solved?

As part of the evidence based medicine process, the development of electronic databases, such as the Cochrane library, should facilitate the process of searching and the critical appraisal of published reports. The Cochrane library is an electronic database which includes systematic reviews with meta-analysis of trials, which have been rigorously evaluated before publication on the Cochrane 
database. The number of systematic reviews or metaanalyses which are applicable to the clinical practice of haematologists is still limited. On the most recent addition of the Cochrane library, issue 4 for 1997, only two systematic reviews could be identified. The controlled trials register within the Cochrane library, however, is more useful. This does include references to randomised controlled trials for areas such as lymphoma, leukaemia, myeloma, and essential thrombocythaemia, and a limited number of trials relative to the management of polycythaemia. This part of the Cochrane library has developed rapidly over the last 12 months and no doubt will continue to do so.

Another approach to the development of evidence based practice is the population adjusted clinical epidemiology (PACE) strategy developed by Proctor and colleagues in Newcastle..$^{90}$ This approach is dependent on collaborative working among a group of haematologists based in the old Northern Region of the National Health Service. This process involves setting up a disease register which collects unselected cases from a demographically defined population. From this database either rigorously controlled or all inclusive studies can then be undertaken. One example of the latter reveals the impact of bone marrow transplantation on an unselected population of young adults with acute myeloid leukaemia. ${ }^{11}$ As mentioned previously the approach taken by PACE includes both the development of and participation in randomised controlled trials and also studies of patients in which evidence from randomised controlled trials does not yet exist. Clearly, for some of the rarer conditions randomised controlled trials may never be developed. The evidence from open studies on these rarer conditions - while at a lower level than that derived from randomised controlled trials-may be the best one can find. This approach also does overcome, to some extent, the barriers to obtaining up to date evidence, since information is provided on a regular basis within the group. This group approach contrasts with the process of evidence based medicine, which is a life long process of self directed learning ${ }^{12}$ focusing on an individual clinician's ability to learn and discover the evidence required to help manage patients.
We have attempted to summarise the degree to which the practice of clinical haematology in the United Kingdom is evidence based. It is clear, however, that for individual haematologists to adopt an evidence based approach to their individual practice there are several significant barriers to be overcome. The collaborative working arrangements which have been developed in the north east of England may be one way of overcoming some of the barriers in introducing evidence based practice into individual hospitals. Despite our long tradition of therapeutic trials in "exciting" diseases like acute leukaemia, the principles and wider practice of evidence based medicine remain a challenge for all haematologists.

M J GALLOWAY

Department of Haematology, Bishop Auckland Hospitals NHS Trust, Bishop Auckland, UK

M M REID

Department of Haematology, Royal Victoria Infirmary and Associated Hospitals NHS Trust, Newcastle upon Tyne, UK

1 Batstone G. Practising by the evidence: the role of pathology. $\mathcal{F}$ Clin Pathol 1997;50:447-9.

2 Fleming KA. Evidence-based pathology. F Pathol 1996;179:127-8.

3 Haynes RB, Sackett DL, Gray JMA, et al. Transferring evidence from research into practice: 1 . The role of clinical care research evidence in clinical decisions. Evidence-Based Medicine 1996;1:7-8.

4 Sackett DL, Richardson WS, Rosenberg W, Haynes RB. Evidence-based medicine: how to practice and teach EBM. London: Churchill Livingstone, 1997.

5 Galloway M, Baird G, Lennard A. Haematologists in district general hospitals practice evidence based medicine. Clin Lab Haematol 1997;19:243-8.

6 Ellis J, Mulligan I, Rowe J, et al. In patient general medicine is evidence Ellis J, Mulligan I, Rowe J, et al.
based. Lancet 1995;346:407-10.

7 Elias L, Portlock CS, Rosenberg SA. Combination chemotherapy of diffuse histocytic lymphoma with cyclophosphamide, adriamycin, vincristine and prednisolone (CHOP). Cancer 1978;42:1705-10

8 George JN, Woolf SH, Raskob GE, et al. Idiopathic thrombocytopenia purpura: a practice guideline developed by explicit methods for the American Society of Hematology. Blood 1996;88:3-40.

9 Charlton BG, Taylor PRA, Proctor SJ. The PACE (population-adjusted clinical epidemiology) strategy: a new approach to multi-centred clinical research. $Q f$ Med 1997;90:147-51.

10 Engels EA, Spitz MR. PACE—-setting research. Lancet 1997;350:677-8.

11 Proctor SJ, Taylor PRA, Stark A, et al. Evaluation of the impact of allogeneic transplant in first remission on an unselected population of patients with acute myeloid leukaemia aged 15-55 years. Leukaemia 1995;9:1246-51.

12 Sackett DL, Haynes RB, Guyatt GH, et al. Clinical epidemiology. A basic science for clinical medicine. Boston: Little, Brown and Co 1991:393-424. 